\title{
Antonio Raimondi y sus vinculaciones con la ciencia europea, 1851-1890
}

Antonio Raimondi et ses liens avec la science européenne, 1851-1890

Antonio Raimondi and his relation with European science, 1851-1890

\section{Lizardo Seiner Lizárraga}

\section{OpenEdition}

Journals

Edición electrónica

URL: http://journals.openedition.org/bifea/6144

DOI: 10.4000/bifea.6144

ISSN: 2076-5827

\section{Editor}

Institut Français d'Études Andines

\section{Edición impresa}

Fecha de publicación: 1 diciembre 2003

Paginación: 517-537

ISSN: 0303-7495

Referencia electrónica

Lizardo Seiner Lizárraga, « Antonio Raimondi y sus vinculaciones con la ciencia europea, 1851-1890 », Bulletin de l'Institut français d'études andines [En línea], 32 (3) | 2003, Publicado el 08 diciembre 2003, consultado el 01 diciembre 2020. URL : http://journals.openedition.org/bifea/6144 ; DOI : https:// doi.org/10.4000/bifea.6144

\section{c) $(7) \Theta$}

Les contenus du Bulletin de l'Institut français d'études andines sont mis à disposition selon les termes de la licence Creative Commons Attribution - Pas d'Utilisation Commerciale - Pas de Modification 4.0 International. 


\title{
ANTONIO RAIMONDI Y SUS VINCULACIONES CON LA CIENCIA EUROPEA, 1851-1890
}

\author{
Lizardo SEINER LIZÁRRAGA*
}

\section{Resumen}

Desde su llegada al Perú en 1850 y por espacio de cuarenta años, el naturalista italiano Antonio Raimondi es considerado uno de los grandes impulsores del desarrollo de las ciencias naturales en el Perú. Viajero incansable, aprovechó cada uno de los recorridos que hizo por espacio de diecinueve años en el Perú para observar una naturaleza deslumbrante que consideraba debía ser conocida en el país y en el extranjero. Su visión de la naturaleza fue totalizadora pues se interesó por todo: rocas, plantas, animales y registros climáticos fueron objeto de observación paciente y acopio sistemático. Raimondi estructuró su contribución al conocimiento de la naturaleza peruana en base a las relaciones que mantuvo con el Estado peruano, su vinculación a las redes profesionales existentes en Lima y, finalmente, su aporte en la difusión de la ciencia contemporánea en el Perú a través de los contactos que estableció con científicos de Europa y América.

Palabras claves: Viajeros, Perú, siglo XIX, historia de la ciencia.

\section{ANTONIO RAIMONDI ET SES LIENS AVEC LA SCIENCE EUROPÉENNE, 1851-1890}

Résumé

Dès son arrivée au Pérou en 1850 et pendant quarante ans, le naturaliste italien Antonio Raimondi est considéré comme un des plus importants moteurs du développement des sciences naturelles au Pérou. Voyageur infatigable il a su profiter de chacun de ses voyages à l'intérieur du Pérou pendant dix neuf ans pour observer une nature excepcionellement riche qu'il pensait devoir être connue tant par les péruviens qu'à l'étranger. Tout lui paraît intéressant: les roches, les plantes, les animaux et plusieurs séquences climatiques furent l'object d'observation patiente et recueillies de façon systématique. Raimondi construisit sa contribution à la connaissance de la nature péruvienne grâce à ses relations avec l'État péruvien, à ses liens avec les réseaux professionnels existant à Lima et aux contacts qu'il établi avec des scientifiques en Europe et en Amérique.

Mots clés : Voyageurs, Pérou, XIX ème siècle, histoire de la science.

* Universidad de Lima. Av. Javier Prado Este s/n, Monterrico. Apartado 852, Lima 100. E-mail: 1seiner@correo.ulima.edu.pe 


\title{
ANTONIO RAIMONDI AND HIS RELATION WITH EUROPEAN SCIENCE, 1851-1890
}

\begin{abstract}
Since his arrival in 1850 in Peru, naturalist Antonio Raimondi was considered as one of the principal driving forces behind the development of natural sciences. Raimondi traveled throughout the country for nineteen years to observe a dazzling nature, which he considers as known in Peru and others countries. His interest embraced almost every aspect of nature: rocks, plants and weather, which were patiently observed on a sistematic basis. Raimondi ellaborated his contribution to the knowledge of Peruvian nature building on his relationships with Peruvian government and professional communities in Lima, as well as his contacts with European and American scientists.
\end{abstract}

Key words: Travellers, Peru, Nineteen-century, History of Science.

\section{INTRODUCCIÓN}

Gracias a las cuatro décadas de vida fecunda dedicada al cultivo y difusión de la ciencia en el siglo XIX, Antonio Raimondi es considerado uno de los hitos de su desarrollo en el Perú. Viajero incansable, aprovechó cada uno de los recorridos que hizo por espacio de diecinueve años en el Perú para observar una naturaleza deslumbrante que consideraba debía ser conocida en el país y en el extranjero. Su visión de la naturaleza fue totalizadora pues se interesó por todo: rocas, plantas, animales y registros climáticos fueron objeto de observación paciente y acopio sistemático. Y aunque la naturaleza lo obsesionó, el hombre no estuvo alejado de sus intereses; desde su admiración por los monumentos incaicos, descritos y dibujados, hasta sus opiniones sobre costumbres y creencias de los pueblos que visitó, todo lleva a considerarlo un observador privilegiado de su tiempo.

El presente trabajo gira sobre tres ejes que revelan la fuerza, magnitud y variedad de las relaciones que estableció el sabio en su estadía en el Perú y que permitieron estructurar su gran contribución al conocimiento de la naturaleza peruana y desarrollo de la ciencia. Tales ejes fueron las relaciones que mantuvo con el Estado peruano, su vinculación a las redes profesionales existentes en Lima y, finalmente, su aporte en la difusión de la ciencia contemporánea en el Perú a través de los contactos que estableció con científicos de Europa y América.

\section{LA VENIDA AL PERÚ: LA RELACIÓN CON EL ESTADO}

Los datos biográficos de Raimondi son bien conocidos. Varias contribuciones han trazado los perfiles centrales de la biografía del sabio italiano (Balta, 1926; Janni, 1942; Azzariti, 2000) aunque es de advertir las marcadas diferencias entre la escasez de datos disponibles para sus primeros años en Italia — los referidos a su infancia y adolescencia - con la abundancia de aquéllos posteriores a su arribo al Perú en 1850. Para esta segunda etapa, su nutrido epistolario, sus propias obras, sus vinculaciones 
profesionales, científicas, amicales y familiares conforman un sólido soporte documental para delinear la vida de la más importante personalidad científica del siglo XIX en el Perú.

Sobre sus primeros veinticinco años de vida, lo único con lo que se cuenta son las propias y escasas referencias de tipo autobiográfico que el mismo Raimondi consignó en su obra. Conocido y repetido es el pasaje donde el sabio refiere el origen de su predilección para elegir el Perú como destino final para emprender el estudio de la naturaleza.

“... Un día estando, como de costumbre, en el conservatorio del Jardín botánico de Milán, presencié por una rara casualidad el corte de un gigantesco Cactus peruvianus, el que habiéndose levantado como un monstruoso candelabro hasta el techo del conservatorio, recorría una gran parte de este, sostenido por medio de cordeles. La mutilación de este patriarca de los cactus, que era una de las plantas de mi predilección, me produjo un vago pesar, como si hubiera sido un ser animado y sensible y esa extraña circunstancia hizo nacer en mí la primera simpatía hacia el Perú, su patria, presagio sin duda de mi futuro viaje a éste país ..." (cursiva nuestra) (Raimondi, 1874-1879, Tomo I: 3) (1).

Poco se ha avanzado para conocer mejor la formación del sabio en Italia. La única fuente hoy disponible para reconstruir dicha época son sus propios recuerdos personales, escritos en el Perú en 1874 como capítulo introductorio a su gran obra, El Perú. Si nos atenemos a lo dicho por él mismo, debió tener acceso a una variada bibliografía de viajeros y científicos. Parte de ella le sirvió para elegir a qué país de América dirigiría sus esfuerzos. Descarta algunos por encontrarse fuera de la zona tropical (Argentina, Uruguay, Paraguay); otros, a pesar de hallarse en dicha zona, los deja de lado por haber sido ya estudiados (Ecuador, Colombia, Venezuela). Con Brasil, “... el primer país que pasó por mi mente ...”, ocurre lo mismo: tropical y sumamente estudiado, a lo que había que sumar el hecho que muchos museos europeos contaban con abundantes colecciones de especies de flora y fauna. Algo semejante le suscita Bolivia “... porque había tenido su explorador en el entusiasta naturalista Alcides d'Orbigny ...". Chile no le llamó la atención pues sumado al hecho de hallarse fuera de su deseada zona tropical, los estudios pioneros de Gay, a los que sumaron los de Philippi y Domeyko — con quienes Raimondi mantuvo posteriormente intercambio epistolar - habían hecho conocido el país en Europa. Ante este panorama, el Perú era una promesa. Si su objetivo fue “... pasar revista a todas las partes de América y escoger la menos conocida y que me proporcionase [...] más abundante cosecha ...", el Perú cumplía tales condiciones pues “... era el país menos conocido hasta hoy ...”. Resulta claro que la elección del Perú como destino de viaje se fundaba en la conjunción del escaso conocimiento de sus producciones naturales en Europa y la altísima probabilidad de acceder a numerosas especies desconocidas para la ciencia. El tiempo le dio la razón.

(1) Varios son los pasajes de este primer tomo en los que el sabio ofrece algunos datos biográficos sobre su estadía europea y de la cual llaman la atención sus constantes visitas a museos, zoológicos y jardines botánicos de todo Europa aunque sin especificar la visita a alguno en particular, salvo al de su natal Milán. 
Si la metódica planificación del viaje de Raimondi al Perú nos es conocida gracias a su propio relato, poca es la exactitud con la que se refiere a sí mismo, pues no aporta fechas ni lugares precisos. De otra parte, la consulta en archivos italianos ha sido prácticamente nula si bien la única pesquisa documental de la que tenemos noticia fue exitosa y estimulante pues permitió rectificar la fecha de nacimiento del sabio, ocurrido en Milán el 24 de septiembre de 1824 (Azzariti, 2000: 20), y así descartar el año tradicionalmente aceptado que se entendía era 1826 (2).

Raimondi intervino activamente en las luchas de emancipación ocurridas en Italia en la década de 1840 y de ello deja prueba el haber participado en las "cinque giornate" ocurridas en Milán en marzo de 1848 (Juarranz de la Fuente, 1984: 33), así como haber combatido en las batallas de Custozza y Novara en julio de ese año y marzo de 1849 (3). Trabajos recientes han demostrado la gran repercusión que dichos acontecimientos tuvieron en la colonia italiana asentada en Lima (Bonfiglio, 1993: 115). Las luchas no alcanzaron la independencia anhelada significando desengaño para quienes participaron en ellas, como fue el caso de Raimondi. Tras su salida de Italia y su embarque en Niza en enero de 1850, Raimondi, junto a otros tres compañeros (4), llegó al Callao el 28 de julio de ese mismo año, según él mismo informa. Llegaba así al país que había elegido aunque sin haber recogido “... cuantos datos me fuera posible sobre la región que iba a visitar y formar un proyecto o plan de estudios ...". Traía cartas de recomendación para el médico Emmanuel Solari —originario de la Liguria, liberal y pariente del patriota Mazini-quien se encontraba radicado en Lima desde 1841, ejerciendo su profesión conjuntamente con la docencia en la única institución peruana dedicada a la formación de médicos en el Perú: el Colegio de la Independencia (5).

La recomendación a Solari fue la vía para entrar en contacto con Cayetano Heredia, director del colegio. A pesar de ser un autodidacta, y en tal medida probablemente no idóneo para el desempeño de una cátedra, Heredia debió entrever capacidades para encargarle el ordenamiento de las colecciones de objetos naturales guardadas en el museo del colegio - al parecer, en atención a sus vastos conocimientos en Ciencias Naturales - labor que realizó satisfactoriamente. Su desempeño en la clasificación de las colecciones debió poner en evidencia su competencia en asuntos científicos y constituyó la razón para que Heredia confiara a Raimondi la cátedra de Historia Natural (Guevara, 1993: 132). Corría 1851 cuando se inició su larga carrera docente, a la que todas las fuentes coinciden en calificar de notable. Dada la escasez de hombres competentes en materias científicas en el Perú de entonces, su labor en el Colegio de la Independencia se tomó en cuenta para que el gobierno del General Echenique lo designara miembro de una comisión formada para calcular las reservas de guano existentes en las islas de Chincha, el mayor depósito de fertilizante en el

(2) Todas las biografías dedicadas al sabio anotan como año de nacimiento 1826 (Vide Balta, 1926; Janni, 1942: 12).

(3) Janni ha trazado un panorama sugerente de esa etapa de la vida del sabio (1942: 22-37).

(4) De los cuatro compañeros de viaje sólo se conoce el nombre de uno: Alessandro Arrigoni, cuya amistad con el sabio se prolongó por décadas. Una aproximación a su vida en: Valdizán, 1924: 111.

(5) Aunque distinto en denominación no se trataba sino del Real Colegio de Medicina de San Fernando, erigido por orden del virrey Abascal en 1808. 
litoral peruano. Lo acompañaría en esta labor otro destacado hombre de ciencia, italiano y también profesor en el mismo colegio, el químico José Eboli (6). A fines de 1853, Raimondi estuvo en las islas por espacio de cuarenta días. Tras emprender trabajos de ubicación de las reservas del fertilizante y analizar su composición, Raimondi y los demás miembros suscriben un informe a fines de noviembre de ese mismo año, calculando las reservas en alrededor de 12 millones de toneladas distribuidas en las tres islas Chincha. Si se le sumaban las existencias anteriormente halladas en la bahía de la Independencia, en la de Ancón y en las islas Lobos “... puede el precioso abono considerarse inagotable durante largos años ...” (Raimondi, 1854) (7). Así cumplía Raimondi el primero de los numerosos encargos que el gobierno iría asignándole a lo largo de las siguientes décadas.

Inmediatamente después, en diciembre del mismo año, Raimondi se encontraba recorriendo la provincia de Tarapacá dedicado a reconocer los yacimientos de salitre, también por encargo del gobierno (1874-1879, Tomo I: 142). En 1856 vuelve a integrar otra comisión, encargada de clasificar las colecciones de piedras, minerales y plantas del antiguo Museo Nacional (Rospigliosi, 1938: 8). A partir de 1858, Raimondi, quien había cumplido cabalmente los encargos asignados y ya había iniciado su larga serie de recorridos por el país (Santillana, 1989) (8), empieza a recibir apoyo económico del gobierno en sus exploraciones. Efectivamente, al año siguiente, el Congreso emite una resolución para que “... se auxilie con dos mil pesos de fondos del tesoro al distinguido naturalista don Antonio Raimondi a fin de que pueda dar la debida extensión al viaje científico que va a emprender por la parte septentrional del país ...” (9). El mismo lo recuerda cuando indica:

“... Todos los gobiernos que se han sucedido desde 1858, ocho años después que comencé mis estudios sobre el Perú, me han prestado directa ó indirectamente su eficaz apoyo, facilitándome el desempeño de la ardua misión que voluntariamente me había impuesto de dar a conocer el país y sus proverbiales riquezas naturales; y lo que es mas, tres Soberanos Congresos me han honrado con resoluciones a mi favor, votándose en 1858 la suma de dos mil pesos y en 1860 la de tres mil para auxiliarme en los crecidos gastos que hacía en mis dilatados viajes por el interior de la república y resolviendo el Congreso de 1869 que la publicación se hiciese a expensas del estado ...” (Raimondi, 1874-1879, Tomo I: III).

Privilegiada fue la situación de Raimondi, pues no conocemos otro caso de viajero o naturalista en el que el gobierno haya invertido cuantiosos recursos en financiar las exploraciones. Sin embargo, el apoyo era merecido. Los viajes de Raimondi

(6) Para una biografía de Eboli consúltese: Valdizán, 1924: 53.

(7) El informe en el que aparece Raimondi se eleva al ministro de Hacienda por carta del 30 de noviembre de 1853. También aparecen como firmantes Francisco Cañas, José Castañón, Fermín Ascencios, José Eboli y Manuel J. San Martín. (pp. 43-50). Sorprende que el nombre de Raimondi no aparezca en otra carta firmada por los mismos miembros con fecha 15 del mismo mes aunque bien podría tratarse de una errata (p. 34).

(8) Se trata de un exhaustivo recuento de todos y cada uno de los viajes realizados por el sabio entre 1851 y junio de 1869 , totalizando 19 años.

(9) Resolución del 21 de marzo de 1859 (Oviedo, 1862, Tomo IX: 155). 
representan un impresionante trabajo de campo en el que el sabio se dedica al acopio y estudio de especies diversas. En ellos se expresa una de las confluencias más significativas entre ciencia y naturaleza ocurridas en el Perú, pues mientras Raimondi aportaba tesón y una curiosidad desbordante, la variedad de la segunda lo fascinó, permitiéndole poner en práctica su andamiaje científico. Por ello es representativa la frase en la que Raimondi condensa la esencia de su experiencia “... me parecía no tener ojos suficientes para mirarlo todo..." (10).

Aunque no haga mención de ella — quizá por representar la ineficacia del Estado en atender prontamente las obligaciones contraídas - una importante norma que lo favoreció fue la resolución del Congreso emitida el 28 de enero de 1866, en virtud de la cual se autorizaba al ejecutivo para asegurar la impresión de su obra y adquirir sus colecciones de objetos naturales, aquéllas que había ido organizando a lo largo de sus viajes y que por su riqueza de especies, constituiría la base sobre la cual se organizaría un nuevo museo dedicado al sabio (Rospigliosi, 1938: 9). Lamentablemente, el Estado tardó varios años en poner en práctica lo acordado. Ni aún con la resolución del 12 abril de 1869 , por la que se fijaba el valor de las colecciones en 50000 pesos, se dio cabal cumplimiento de lo estipulado. Debieron pasar otros años hasta que, según decreto del 20 de junio de 1873 , se daba cumplimiento a lo ordenado en 1869 . Tras siete años transcurridos esperando la materialización de un compromiso formal, Raimondi no podía sino reconocer que “... estaba reservado al ilustrado gobierno del Excmo. Señor D. Manuel Pardo, el dar la más decidida protección a esta obra ..." (Raimondi, 1874-1879, Tomo I: III). Al año siguiente salía publicado el primer tomo de El Perú, síntesis de los largos recorridos que habían llevado al sabio a visitar casi todo el territorio. Antes de concluir la década de 1870, habían visto ya la luz dos tomos más. Grande fue la estima profesada por Raimondi al presidente Pardo y de ello queda puntual testimonio en las numerosas cartas que ambos se dirigieron y que felizmente se conservan (11).

Finalmente, transcurridos los años de la Guerra, en 1886, el Congreso, por ley del 14 de octubre, dispuso la continuación de la obra El Perú (La Torre, 2000: 217), decisión que no llegó a materializarse pues Raimondi no alcanzó a ver en vida un

(10) Una apretada síntesis de sus viajes la ofrece en el primer tomo de El Perú. Entre 1851 y 1858 realiza sus primeros viajes, nueve en total. A partir del 26 de marzo de 1859 y hasta 1861 , emprende su primer gran viaje al norte y a la región del Amazonas, demandándole veintitrés meses. Luego, tras realizar algunos viajes cortos por espacio de mes y medio en los alrededores de Lima, a partir de mayo de 1862 inicia su viaje general por el sur de la república, interrumpido cinco meses debido a una dolencia que se le manifestó estando en el mineral de Quispisiza. Recuperado, parte nuevamente de Lima en agosto de 1863, continuando el viaje en dirección a Acarí y de ahí hacia Lucanas y luego hacia la costa nuevamente. 2500 leguas recorridas, aproximadamente 12000 kilómetros, en dos años y medio. Incansable, emprende un viaje de cuatro meses por el centro del país. Finalmente, desde junio de 1867 se inicia el último gran viaje, esta vez, regresando al norte y ampliando el recorrido hacia el centro. Tras 19 años de viajes constantes, constituyendo una empresa individual única y no repetida, él mismo anotaba su "... ingreso a Lima el 10 de junio de 1869, dos años después de mi salida ..." (Raimondi, 1874-1879, Tomo I: 418).

(11) A título de ejemplo puede apreciarse la confianza de Pardo en Raimondi cuando le escribe pidiéndole le asesore en relación a los misioneros que exploraron y colonizaron la zona de Chanchamayo. Ver: Antonio Raimondi a Miguel Colunga. Huaraz, 7 enero 1867, carta reproducida (Raimondi, 1991: 175). 
nuevo tomo de su obra (12). En las cartas de aquella época se trasluce su desazón, dudas y enojos por las sucesivas dilaciones en las que el gobierno incurría. Los achaques y el desánimo se fueron apoderando del otrora vigoroso científico. La muerte lo sorprendió en San Pedro de Lloc - localidad ubicada en las sierra norte del país-en octubre de 1890 (Janni, 1942: 316).

En su relación con el Estado, no puede soslayarse los veinte años que Raimondi desempeñó de forma ininterrumpida su labor docente en el Colegio de la Independencia, así como en las facultades de Medicina y Ciencias de la Universidad Mayor de San Marcos. Una mirada de conjunto sobre dicha labor la hemos planteado con anterioridad (Seiner, 2003).

Paralela a su labor docente el sabio cumplía otras actividades. A partir de la década de 1850, sus pareceres técnicos eran solicitados frecuentemente por numerosos funcionarios públicos. Un artículo pionero, basado en su visita a Chincha en 1853 y publicado en los Comptes Rendus de l'Académie des Sciences de Paris en 1856, daba cuenta del origen del fertilizante, descartando que se trate de un coprolito, como era común creencia en Europa (Raimondi, 1856). En 1873 planteaba la modificación de la composición del guano extraído de las islas Guañape, considerado de baja calidad por su baja concentración de amoniaco. Por el contrario, el guano de alta calidad, aquél que contiene exceso de amoniaco, superaba con creces las necesidades de la agricultura. El objetivo de Raimondi era ofrecerle al gobierno parámetros técnicos para negociar con los comerciantes y no ceder a sus exigencias. Así se establecerían expectativas aceptables y sensatas de ganancia que debía tener el gobierno en relación con la comercialización del producto (Raimondi, 1973b: 7). La opinión de Raimondi se originaba en la necesidad de aclarar y poner en su justa medida las opiniones emitidas por Daniel Ruzo, antiguo delegado fiscal del Estado en el negocio del guano, quien planteaba que añadiendo al producto ciertas sustancias químicas podían obtenerse elevadísimas ganancias, lo que el sabio consideraba ilusorio (Raimondi, 1973c) (13). La sustentación de dichas opiniones le valió ser muchas veces atacado por rivales de ocasión y en sus réplicas ganó fama de acerado polemista.

Desde 1861 se desempeña, oficial e ininterrumpidamente, como geólogo y químico consultor del Estado peruano. Tras los años aciagos de la guerra en los cuales se dedicó a su labor como ensayador, cumpliendo encargos que le confiaban particulares en la capital (Llona, 1884: 29), el gobierno lo vuelve a nombrar en su cargo de geólogo consultor del Estado con el que solía presentarse (Raimondi, 1990: 52). Los sueldos atrasados son testimonio de las difíciles condiciones a las que el sabio debió enfrentarse (La Torre, 2000: 215). Osciló así entre los empleos del gobierno y el ejercicio de su oficio a favor de particulares. Hacia 1885, sus

(12) En los años siguientes, instituciones como la Sociedad Geográfica de Lima y el Cuerpo de Ingenieros de Minas publicaron tres tomos más bajo el título El Perú. Martín Ueda ha aclarado las vicisitudes que enfrentó la publicación de los tomos encargados a la segunda de las mencionadas. Véase Ueda, 2002: 216.

(13) Pareciera tratarse del mismo texto mencionado en la cita anterior pero en formato mayor aunque faltan verificar las semejanzas y cual de los dos efectivamente se publicó primero. La única diferencia advertible de primera vista es una carta enviada por Raimondi que obra en éste. 
obligaciones como consultor del Estado le exigieron total dedicación. Por ese entonces, Raimondi recibía su sueldo de la Escuela de Ingenieros, la cual financiaba la impresión de su obra y la entrega de sus sueldos gracias a la recepción del impuesto de minas (Raimondi, 1990: 53; López Soria, 1999) (14).

El Estado le asignó varias membresías. Las más importantes fueron su designación en 1888 como socio activo de la recién creada Sociedad Geográfica de Lima (La Torre, 2000: 219) (15) y de la Sociedad de Agricultura y Minería ese mismo año. Años antes, en 1876, se le nombra para formar parte del consejo editorial de una nueva publicación especializada perteneciente a la novel Escuela de Ingenieros, los “Anales de Construcciones Civiles y de Minas del Perú" (Guevara, 1993: 133) (16), la que aparecería recién en 1880 y en la que publicó varios artículos. Para las nuevas instituciones, contar con un miembro de la talla de Raimondi ciertamente contribuía a proyectar una imagen de seriedad y rigor científico (Raimondi, 1990: 63).

Los compromisos de Raimondi con el Estado también incluían la promoción del país en el extranjero. Tal fue el carácter del compromiso contraído en 1878 con el gobierno del General Mariano I. Prado para preparar una colección de minerales a exhibirse en la Exposición Universal de París de ese año (Raimondi, 1990: 42). La muestra incluyó varios cientos de minerales y formó parte del Grupo V, clase 43 “... Produits d' exploitation des mines et métalurgie ...” (Abertini, 1878: 44) (17), que fue premiada con una medalla. La Exposición de París fue también ocasión para que Raimondi difundiese su propia obra: los dos primeros tomos de El Perú se exhibieron en dicha ocasión y creemos que contribuyeron a fortalecer la imagen de reputado naturalista que había venido creando en Francia desde sus pioneros artículos sobre el guano publicados hacía veinte años.

En suma, la relación de Raimondi con el Estado peruano fue larga y provechosa pero jalonada de hechos sucesivos que, aunque lo mantuvieron con frecuencia en situaciones de estrechez económica, finalmente arrojó un balance positivo, expresado en el apoyo a sus viajes, la publicación de su obra y la confianza depositada en sus dotes profesionales.

\section{RAIMONDI Y LAS REDES CIENTÍFICAS NACIONALES: MÉDICOS E INGENIEROS}

En sus recuerdos de juventud como escolar en el Colegio de San Carlos allá por 1850, Ricardo Palma aludía a la amena tertulia que congregaba a un grupo de jóvenes

(14) López Soria incluye el presupuesto de la Escuela de Ingenieros entre 1881 y 1909 (p. 49).

(15) Para la relación de socios fundadores, véase: Palacios, 2002: 37.

(16) Hace referencia a la resolución suprema de 3 de julio de dicho año.

(17) El catálogo al que hace referencia aquí y que se publicó aparte es: Minerales del Perú o catálogo razonado de una colección que representa los principales tipos minerales de la república, con muestras de huano y restos de aves que lo han producido (1878). Jean-Baptiste Martinet, comisario de la exposición peruana en París lo tradujo al francés como: Minéraux du Pérou. Catalogue raisonné d'une collection des principaux types minéraux de la République comprenant aussi des échantillons de guano et des derbis fossilisés des oiseaux qui l'ont produit (1878). 
en la habitación de uno de los estudiantes de medicina del Colegio de la Independencia. Uno de aquellos días “... apenas transcurridos seis u ocho días (del 28 de julio) intimamos con el joven italiano, intimidad inalterable hasta el año en que Raimondi realizó su primer viaje de exploración a la montaña ..." (La Torre, 2000: 206) (18). Tal fue el primer vínculo amical que Raimondi estableció en el Perú, sobre el cual se fue construyendo una red amplia de amistades. En las cartas dirigidas a su amigo Colunga a comienzos de la década de 1860, refiere la existencia de la Ilustrada Sociedad Pitológica (Raimondi, 1991: 33), mención que daba al grupo de amigos que tenía en Lima y con los que se reunía cada vez que volvía a la capital tras cada viaje. Con ellos mantenía charlas amenas en las que no faltaba ni el café ni el cigarrillo, que eran moneda común de los "pitólogos". De hecho, por su esencia eminentemente amical, dicho grupo distaba de ostentar el carácter oficial de la Sociedad Filotécnica, entidad académica apoyada por el gobierno que fundó con su gran amigo, el poeta romántico Manuel Nicolás Corpancho (Janni, 1942: 91, 107). Dicha entidad se fundó el 27 de junio de 1856 y estaba formada por socios profesores que asumían la responsabilidad de impartir lecciones dominicales de Ciencias y Matemáticas al público interesado (Fuentes, 1858: 256). Desconocemos el tiempo por el que se mantuvo vigente dicha asociación y si llegó a ser contemporánea de la Sociedad Amantes del Saber, que vio la luz en Lima el 16 de enero de 1870 y entre cuyos fundadores se hallaban José Vicente Oyague y Soyer y el italiano Giovanni Capelo. Uno de los propósitos que los animaba fue formar bibliotecas y gabinetes necesarios para el progreso de las ciencias matemáticas, naturales y morales (19). Ya Marcos Cueto llamó la atención sobre la innovadora perspectiva que dicha asociación introdujo en el mundo intelectual limeño, tan poco afecto a asuntos relacionados con el cultivo de las ciencias naturales (Cueto, 1993: 46).

En pocos años, Raimondi se encontraba inserto en los círculos intelectuales y profesionales de Lima. Mariano Felipe Paz Soldán, Nicolás Fernández de Piérola - padre del futuro caudillo y presidente y a quien le dedica una encomiástica reseña en la que lo califica de "gloria peruana olvidada"- y el mismo Heredia se contaban entre las amistades del sabio. A estos se sumaban los médicos, especialmente italianos en un primer momento “... con quienes era mayor para Raimondi la afinidad de cultura ..." y que incluía a los doctores Solari, Giovanni Capello y Giuseppe Caffari (Bonfiglio, 1993: 70). Los médicos fueron la primera red a la que se vinculó Raimondi. Primero italianos y luego conjuntamente con los nacionales, conformaron un importante referente amical y de conocimientos para el sabio.

No sólo el hecho de encontrarse vinculado a la Escuela de Medicina inducía las investigaciones de Raimondi. Sus estudios sobre aguas potables y minerales estaban impelidas por el afán de hacer conocer sus virtudes curativas a los médicos. En un trabajo dedicado al análisis de las aguas minerales de Arequipa publicado en 1864 indica que los resultados servirán “... al cuerpo médico del Perú para que prescriba su uso en la curación de enfermedades ...” (Raimondi, 1864: 3). Al presentar el análisis de

(18) Se extrae la información del discurso pronunciado por Ricardo Palma con ocasión de inaugurarse el monumento del sabio en 1910. Véase: Palma, 1910.

(19) El Siglo, periódico científico y literario (Lima). $N^{\circ} 1,1$ de noviembre de 1874. 
las aguas de la fuente de Yura, dividiéndolas en sulfurosas y ferruginosas, recomienda el uso de las primeras para la curación de enfermedades cutáneas — dada su mayor concentración de gas sulfhídrico- mientras que las otras servirían para combatir el reumatismo debido a la mayor presencia de gas carbónico (Raimondi, 1864: 4).

Dos fueron las generaciones de médicos con los que Raimondi tomó contacto: la primera, la de Heredia, Miguel de los Ríos y Solari y otra nueva, la de Rosas, Ulloa, Pro y Bambarén, a quienes tuvo originalmente como discípulos. La estadía de éstos en París, gracias a la generosidad de Heredia, le facilitó a Raimondi recibir bibliografía actualizada e instrumentos para los gabinetes del Colegio de la Independencia (Basadre, 1983, Tomo IV: 310). Aunque no exista la misma disponibilidad de fuentes que en el caso de los médicos, no es desdeñable la vinculación del sabio con los farmacéuticos de la capital, pues publicó diversos artículos científicos en los Anales de la Sociedad de Farmacia, entidad organizada en Lima en 1871 (20).

La otra gran red a la que Raimondi se vinculó fue la de los ingenieros, constituida con posterioridad a la de los médicos pues sólo a comienzos de la década de 1850 se crea la Comisión Central de Ingenieros Civiles (López Soria \& Cazorla, 1999:14). La expansión de ambas coincide con el período de prosperidad falaz por el que atraviesa el Perú gracias a la riqueza del guano (Cueto, 1993: 45). Entre los ingenieros, Raimondi trabó particular amistad con Ernesto Malinowski, polaco de nacimiento. En su último viaje, que lo llevó por la selva norte y le demandó dos años continuos, pasando por el Callejón de Huaylas y llegando a Yungay “... esperaba de día en día el regreso del ingeniero en jefe, Señor Malinowski, con quien había convenido ir juntos a Lima ..." (Raimondi, 1874-1879, Tomo I: 418). En 1869, el gobierno había reconocido a Enrique Meiggs como concesionario de la construcción del ferrocarril Chimbote-Huaraz quien a su vez nombró a Malinowski para emprender los estudios definitivos (López Soria, \& Cazorla, 1999: 26). El regreso a Lima con Malinowski debió resultarle gratísimo a Raimondi pues a lo largo de sus viajes, Colunga y el ingeniero polaco eran quienes más le escribían, asunto importante para una persona sensible como el sabio para quien “... un pobre hombre que viaja solo se consuela mucho cuando recibe alguna carta de los amigos y le parece estar entre ellos ..." (Raimondi, 1991: 35). La mutua estima que existió entre ambos se vio fortalecida cuando Raimondi elige a Malinowski como su testigo de matrimonio (Janni, 1942: 259). Inclusive, una de las últimas cartas que Raimondi escribe desde San Pedro de Lloc, pocos meses antes de su deceso, tiene como destinatario al ingeniero polaco (21).

Malinowski fue el puente de contacto con Meiggs, quien estuvo interesado en la publicación de los escritos de Raimondi sobre el departamento de Ancash, pues sabía que las riquezas naturales que el sabio descubría y estudiaba serían un poderoso acicate para estimular su explotación utilizando el ferrocarril. Tal interés explica el porqué Meiggs financió, en 1873, una de las principales obras de Raimondi, El departamento de Ancachs (Raimondi, 1873a). Teniendo en cuenta los jugosos beneficios

(20) Artículos de 1868 y 1873 se incluyeron en los anales publicados por dicha Sociedad. Véase, Balta, 1899: 5.

(21) Raimondi a Malinowski. San Pedro, junio 27, 1890 (Raimondi, 1990: 77). 
financieros recibidos por el empresario norteamericano a raíz de la larga serie de obras públicas que le confiaba el gobierno — situación que ha recibido severos juicios (22) es menester interrogarse sobre el modo en que se plantearon las relaciones entre ambos, toda vez que la acrisolada moralidad de Raimondi debió maliciar las intenciones de Meiggs y, en consecuencia, entre ambos no debió existir sino una respetuosa relación, estrictamente comercial.

Al final de su vida, Raimondi había formado un solo núcleo con profesionales de ambas clases al que se habían ya unido literatos y periodistas. El testimonio de Aurelio Arnao, sobrino del sabio, es elocuente de ello. Indicaba cómo, hacia 1886:

“... A las tres de la tarde tomaba café generalmente acompañado de algunos amigos, siendo el encargado de preparar la bebida aromática (café) el políglota austriaco don David Pretzner. Entre sus contertulios (...) recuerdo a los doctores Colunga, Ulloa, Bambarén, Habich, Malinowski, Carranza, Chiarella, García Merino, Emiliano Llona, el viejo aristócrata romano Carenci Gallezzi (...) Juan de Arona, Ríos, Remy y otros ... (cursiva nuestra)" (La Torre, 2000: 218).

La relación que Raimondi mantuvo con el Estado, su práctica docente y el carácter de sus vínculos amicales, que proveyeron al sabio de crítica y consejo, determinó que la figura de Raimondi fuera cada vez más influyente en diversos círculos científicos, políticos y sociales. Este contexto contribuyó a apuntalar la dimensión de Raimondi como intermediario científico, promoviendo el intercambio de conocimientos con pares en Europa y América.

\section{RAIMONDI Y LA DIFUSIÓN DE LA CIENCIA EUROPEA EN EL PERÚ}

A pesar de su volumen — tres tomos con más de 1000 páginas-El Perú, la obra querida del sabio, publicada en vida y dedicada a la juventud peruana, es una apretada síntesis de la obra raimondiana. En el tomo primero, denominado Parte preliminar, el viajero y naturalista sintetiza los veinte años de viajes emprendidos en toda la extensión de la república. Por su parte, los tomos segundo y tercero se abocan a la presentación de una lograda Historia de la Geografía del Perú (23). En conjunto, los tres tomos se conciben

(22) Basadre, 1983, Tomo V: 127-128, para una sólida crítica a las especulaciones financieras de Meiggs.

(23) Una primera aproximación a El Perú nos revelaría una sencilla división terciaria en tomos, libros y capítulos. Los tres tomos fueron publicados a lo largo de la década de 1870 en la imprenta del Estado gracias al apoyo del gobierno de los presidentes Pardo y Prado. En la Historia de la Geografía del Perú, que ocupa la totalidad de los tomos segundo y tercero (Lima, 1876; 1879), Raimondi emprende una exhaustiva revisión de todos los autores que se ocuparon del territorio peruano entre los siglos XVI y XIX. Esta sección la denomina Historia de la Geografía del Perú o relación cronológica de los viajes, descubrimientos, fundaciones de ciudades y pueblos, cambios notables de las divisiones territoriales que se verificaron en el Perú desde 1801 hasta el día. Este libro, a su vez, se divide en otros dos libros (sic) que cubren la totalidad del tomo tercero. El primer tomo se halla dividido en dos libros: el primero se titula Libro I, Origen de mis estudios sobre el Perú, breve relación de los anteriores, modo como se han hecho para la presente obra y guía para los que deseen continuarlos y comprende las primeras 137 páginas mientras que el segundo es Libro II, Viajes emprendidos en todo el territorio de la república para recoger el material y datos para la obra "El 
como parte de una obra de mayor volumen que Raimondi imaginó y deseó publicar en vida pero que su muerte truncó parcialmente. Su gran proyecto editorial, que comprendía no menos de veinte volúmenes, hubiese significado el summum de la ciencia peruana del siglo XIX. Una obra de semejante envergadura debió imaginarla Raimondi bajo la influencia de la obra de Humboldt, vastísima y dedicada al análisis del Caribe y parte de América del Sur, en la que el Perú recibe un tratamiento más bien marginal. Al momento de su arribo al Perú, todavía se seguía publicando en Europa la magna obra de Humboldt y se hallaba aún en vida, el grueso del Viaje a las Regiones Equinocciales se hallaba ya publicado (Humboldt, 1814-1831) y Raimondi conocía su obra. Así lo señala cuando afirma que:

“... Nacido con una decidida inclinación a los viajes y al estudio de las ciencias naturales, soñé desde mi infancia con las espléndidas regiones de la zona tórrida. Más tarde con la lectura de varias obras de viajes, tales como las de Colón, Cook, Bougainville, Humboldt, Dumont d'Urville despertaron en mí el mas vivo deseo de conocer aquellas comarcas privilegiadas ..." (Raimondi, 1874-1879, Tomo I: 1).

Al observar el plan general de la obra de Humboldt no puede dejar de constatarse las semejanzas con el proyecto editorial de Raimondi. Seis partes conformaban el plan general que Humboldt había adoptado tras su regreso de América en 1804: Física General y Relación del Viaje, Zoología, Astronomía y Magnetismo, Geología, Botánica y una sección exclusiva dedicada a México. Sólo la parte dedicada a la narración del viaje comprendió seis volúmenes en tres tomos publicados en París y Londres entre 1814 y 1831. Toda la obra de Humboldt abarcó 30 tomos de gran formato que incluían mapas y láminas sobre los distintos órdenes de la naturaleza (Minguet, 1980). Varios factores confluyeron en la materialización de la obra del prusiano: aparte de su reconocidísimo talento y el acopio masivo de información sobre la naturaleza americana, su sólida formación en ciencias, su longevidad - llegó a los 90 años_ - y la disponibilidad de una cuantiosa fortuna personal que prácticamente invirtió en su totalidad en la publicación de su obra, dieron cima a su proyecto. En Raimondi, por el contrario, no hallamos tal confluencia. Raimondi y Humboldt percibieron la naturaleza como una totalidad y su comprensión también debía ser totalizadora; por ello, no desdeñan ningún aspecto de su estudio. El sueño de abarcar en una obra lo que su experiencia directa les ofreció los motivaba a publicar y hacer conocidos a otros territorios sobre los que pendían, generalmente, visiones excéntricas e inexactas. Raimondi ni recibió herencia alguna ni dedicó su vida a labrar fortuna. Sin ser precaria, la vida de Raimondi se vio a veces sumida en estrecheces a fin de enfrentar las responsabilidades derivadas de la educación de sus menores hijos (24), el deterioro en la salud de su esposa (Janni, 1942: 272 y ss) y los achaques que él mismo padecía (25).

Perú" abarcando las siguientes 300 páginas. Por lo tanto existe una doble división en libros que podría llevar a confusión en el contexto general de la obra.

(24) Tres fueron los hijos del matrimonio de Raimondi con doña Adela Loli, celebrado en Huaraz el 2 de setiembre de 1869, coincidentemente cuando el sabio había terminado sus largas travesías por el Perú (Janni, 1942: 271). Fueron éstos Enrique, María y Elvira.

(25) Desde inicios de la década de 1870, los males estomacales lo aquejaban frecuentemente tal como se desprende de la lectura de las varias cartas que el sabio envió, entre 1873 y 1876, al presidente Manuel Pardo (Ver: AGN. Sección Republicana, Cartas a Manuel Pardo). 
No tuvo una vida longeva — falleció a los 66 años — ni podríamos aún establecer en qué medida pudo superar su condición de autodidacta, aunque el volumen de lo acopiado en sus viajes, hechos en veinte años frente a los cinco de Humboldt en América, hacen fuertemente respetable su contribución a la ciencia. Aunque no sea lo más conveniente evaluar tan puntualmente vidas que jamás se cruzaron, sorprende el volumen de la obra publicada e inédita de Raimondi y más aún realizada en las condiciones descritas. Balances hechos a su muerte por comisiones ad hoc nombradas por el gobierno muestran la monumentalidad de su aporte: una vasta producción bibliográfica conformada por libros, folletos y artículos publicados en diarios y revistas científicas del Perú y el extranjero que suman poco más de 60 publicados en vida (Balta, 1899: 2-9), decenas de libretas y cuadernos de apuntes parcialmente publicados — de desigual calidad en la edición (26)—, centenas de observaciones meteorológicas, un mapa completo del Perú y varios parciales y abundantes colecciones que sumaban casi 3000 muestras minerales, 2000 fósiles, 700 rocas, 20000 plantas, casi 1300 aves, 4000 insectos y 2000 moluscos yomo para no dudar de la gran y totalizante mirada que el sabio dio al Perú y en la que ciertamente incluía la presencia del hombre, más de 100 cráneos (Azzariti, 2000: 9), probablemente recogidos en yacimientos arqueológicos. En suma, un verdadero arsenal para emprender un estudio exhaustivo de la naturaleza en el Perú. Los hombres de ciencia así lo reconocen. Uno de ellos afirmó que “... sus trabajos constituyen la más extraordinaria fuente de información con que puedan contar nuestros científicos ..." (Guevara, 1993: 128). Si la conformación de colecciones significaba un directo impulso al desarrollo de la ciencia, también ello representaba una forma de dar a conocer el país en el extranjero.

De ello queda evidencia reveladora. En la introducción a su Catálogo razonado... Raimondi justifica la inclusión del mismo como parte de la colección de minerales recogidos en el Perú y enviada a París con ocasión de la Exposición Universal de 1878 con el siguiente argumento: “... muchos minerales son enteramente desconocidos en la ciencia y no podrían a la simple vista, en una exposición, ser reconocidos ...”. En una época donde las ciencias naturales quieren dar cuenta de la diversidad de manifestaciones de la naturaleza a lo largo del mundo, un naturalista de la seriedad de Raimondi significaba para la ciencia europea la certeza de aumentar considerablemente la oferta de especies desconocidas. Sus tempranas publicaciones en prestigiosas revistas científicas europeas le fueron ganando fama de seriedad. Por ejemplo, desde Humboldt y Rivero nadie había descrito el guano de la manera que el sabio lo hizo. Trasluce a lo largo de su obra su afán por dar a conocer las riquezas naturales del país, aquéllas que él mismo tuvo ocasión de acopiar durante sus viajes. Como gran y meritísimo difusor de las riquezas del país, pocos hicieron tanto por apuntalar la imagen de un país ubérrimo en recursos naturales.

Aparte de sus conexiones con los más importantes representantes de la élite profesional de Lima, Raimondi tuvo activa vinculación con instituciones científicas extranjeras. Desde los primeros artículos que fueron publicados en los prestigiosos Comptes Rendus de l'Académie des Sciences de Paris en 1856 y los que se incluyeron

(26) Marcos Cueto ya ha descrito los principales repositorios de Lima donde es posible encontrar documentación sobre Raimondi. Véase Cueto, 1995: 174-178. 
en el Journal of the Royal Geographical Society de Londres (Balta, 1899: 4-5), Raimondi fue conocido por numerosos científicos del exterior y mantuvo correspondencia con ellos. Tal fue el caso, en América del Sur, de Rudolph Amandus Philippi, director del Museo de Historia Natural de Santiago de Chile.

Alemán de nacimiento, Philippi había llegado a Chile en 1851 y tras unos inicios difíciles fue invitado por el gobierno de Manuel Montt para hacerse cargo de la dirección del Museo de Historia Natural, fundado por Claude Gay, científico francés, en 1830. Formado en medicina y ciencias naturales, su presencia en Chile representó un decisiva contribución al desarrollo de las ciencias naturales. Paralelamente a su cargo en el museo, Philippi se dedicó a la enseñanza en la Universidad de Chile y fue consultor técnico del gobierno en materia de producciones naturales. Decenas de viajes le sirvieron para escribir una larga serie de artículos científicos sobre flora, fauna y minería (27). En un artículo temprano, Philippi informa sobre sus hallazgos de nuevas especies de pájaros y aves (Philippi, 1857). Una personalidad de tal calibre llamó la atención del milanés. Raimondi y Philippi mantuvieron estrecha colaboración científica aparte de una mutua admiración. Para el alemán significaba un asunto importante conocer personalmente al italiano y así se lo comunica (28).

El estudio de moluscos, uno de los tantos intereses de Philippi en materia zoológica, se convirtió en el punto inicial de contacto. Para Raimondi, una figura como Philippi era el asidero necesario para emprender la clasificación de los ejemplares que le había enviado; su opinión era necesaria a fin de establecer si dichas especies eran efectivamente desconocidas para la ciencia. Inclusive, a fin de establecer más fidedignamente su valor, Philippi había tenido la iniciativa de enviar algunas a Europa para recibir opinión de otros especialistas.

La circulación de las especies recogidas y enviadas por Raimondi revela una estrategia científica que ayuda a entrever el establecimiento de eficaces redes con el exterior que en el caso de los moluscos estaría evidenciando una suerte de “cuadrangulación". Deseoso de alentar contactos, Raimondi envía simultáneamente colecciones de moluscos a Philippi y a un desconocido científico inglés a quien le comunica en 1867 que "... los pocos ejemplares que le mando a usted son los mismos de los que envié al señor Philippi ...” sugiriéndole que tuviese a bien “... para evitar confusiones creando otros nombres, puede usted escribir mandándole una lista de sus números al Dr. Armando Philippi, a fin de que le mande los nombres que ha dado ..." (29). De paso, le informaba al mismo destinatario que “... hace poco recibí una carta en la que me decía [Philippi] que había terminado la clasificación, pero que para hacer un trabajo más exacto, había enviado a Alemania las especies nuevas y dudosas al célebre helicógrafo [sic] Pteitler para obtener su opinión ...” (Raimondi, 1990: 19). Raimondi debió seguir enviando ejemplares de moluscos a Philippi pues este le indica en carta de

(27) Schell, Patience A: “The Philippi Family in Chile”. Véase: Relics and Selves (interactive exhibition) Iberoamerican Museum of Visual Culture on the web. Birkbeck College. University of London. http://www.bbk.ac.uk/ibamuseum/texts/Schell04A.htm

(28) Museo Antonio Raimondi (Lima). Archivo Raimondi. Epistolario. Rudolf Amandus Philippi a Antonio Raimondi. Santiago de Chile, agosto $1^{\circ}, 1869$.

(29) Antonio Raimondi. Lima,8 de mayo de 1867 (Raimondi, 1990: 19). 
julio de 1870 que había tenido demoras con la que suponemos era una segunda clasificación de muestras (30).

Cuatro científicos involucrados revelan a través de sus contactos el proceso de acumulación de ejemplares que permitía adelantar en el conocimiento de una rama específica de las ciencias naturales. Raimondi debió ser consciente del rol que cumplía en la red. Aún cuando sabía que era el eslabón inicial de la cadena en su condición de acopiador/aportador de especies y que en el desempeño de tal rol era enorme su contribución a la ciencia, también aspiraba a conocer los resultados de la clasificación que los demás habían realizado. Su opinión es clara al respecto al comunicarle al mismo científico inglés citado líneas arriba “... suplico a usted que me haga saber el nombre que adopten y la obra a donde se publique su descripción ...”. Nada más alejado de la realidad que entender a Raimondi como un pasivo y dadivoso aportador de especies. Para el sabio, un envío de muestras al exterior abría la posibilidad de obtener reales beneficios. ¿Qué le reportaba a Raimondi enviar especies al exterior? Más allá de hacerse conocido y aceptar membresías en sociedades científicas, un envío de especies al exterior podía significar, en reciprocidad, tener acceso a bibliografía actualizada. No deja duda el tenor de las siguientes palabras “... como yo me ocupo solamente del estudio de todas las producciones naturales del Perú, no me interesan los moluscos de otro país y más bien desearía conseguir obras que traten de este ramo, de consiguiente suplico a usted que en cambio de los moluscos que le envío me mande alguna obra que sirva para su clasificación. En caso de que la obra tenga mayor valor, a mi regreso a Lima le enviaré otra colección... [cursiva nuestra]”. Así como explicita sus intereses, también se siente comprometido con quienes le envían bibliografía sin haberla solicitado. En una de las tantas cartas enviadas a Miguel Colunga durante sus travesías, Raimondi le cuenta cómo “... vi con placer el regalo de libros hechos por el señor Müeller, director del Jardín Botánico de Melbourne pero no se qué le podemos mandar a cambio [...] sería bueno hacer recoger algunas semillas de plantas indígenas aunque sea de los alrededores de Lima y yo veré si puedo recoger algo en mis excursiones ...." (31). Como científico, a Raimondi le interesó siempre estar actualizado y en virtud de ello el acceso a la bibliografía constituyó una preocupación permanente. El establecimiento y mantenimiento de redes de contacto con científicos del exterior significó para Raimondi implementar un eficaz nivel de reciprocidad en el que el envío constante de especies era la garantía de inserción a dicha red, la que a su vez actuaba como un canal a través del cual tuvo acceso a información actualizada.

Los moluscos constituían sólo una parte de los variados intereses que le suscitaban la observación y el estudio de la naturaleza. Apréciese en la siguiente cita la claridad con la que Raimondi los deja entrever claramente, afirmando que “... en mis viajes me ocupo de todos los ramos de las ciencias naturales [por lo tanto] no puedo dedicarme exclusivamente a los moluscos, y por consiguiente, me falta tiempo para buscarlos ...". De cada viaje, Raimondi llevaba ejemplares al gabinete de Historia Natural que existía en la Facultad de Medicina de San Fernando en Lima. Sin embargo, no todos llegaban

(30) Museo Antonio Raimondi (Lima). Archivo Raimondi. Epistolario. Rudolf Amandus Philippi a Antonio Raimondi. Santiago de Chile, julio 16, 1870.

(31) Raimondi a Colunga. Huacho, octubre 5, 1867 (Raimondi, 1991: 161). 
a la ciudad. Sabedor de la duración aproximada que cada viaje al interior del país le demandaría, Raimondi aprovechaba para enviar muestras al exterior desde distintos puntos de su itinerario.

Tan nítida como la relación sostenida con Philippi, también lo fue la que mantuvo con Constantin Jelski, ornitólogo polaco. Nacido en Lituania en 1837 en una próspera familia de agricultores, Jelski estudió en Minsk para luego dirigirse a Moscú donde dejó inconclusos sus estudios de medicina, pues fue absorbido por sus estudios de ciencias naturales en Kiev. A pesar de ofrecérsele la cátedra de zoología en la Universidad de Kiev, prefirió partir a Turquía donde llevó a cabo investigaciones geológicas por cuenta del gobierno turco durante dos años ([Kochanek], 1979: 104). En 1865 viaja de Turquía a Francia desde donde parte a la Guyana Francesa tras haber tomado contacto con el laboratorio zoológico del profesor Deroylle.

La estadía de Jelski en la Guyana se prolongó por espacio de cuatro años en los que realizó una labor diversa. Obtuvo un empleo de aprendiz farmacéutico en el hospital de Cayena que le permitió dedicarse a la docencia y a realizar recorridos por las inmediaciones de la ciudad a fin de recoger muestras que enviaba a Europa; en los primeros años, colecciones enteras de orquídeas llegaron a Varsovia y otras tantas de aves a París. A partir de 1868, los envíos se canalizan básicamente a Varsovia. Gracias al contacto obtenido con un noble polaco protector de las ciencias naturales, el Conde Branicki, Jelski aseguró el financiamiento de sus expediciones a cambio del suministro de ejemplares de flora y fauna al Gabinete Zoológico de Varsovia ([Kochanek], 1979: 107), hoy Museo de Historia Natural de la ciudad. Debido a problemas de salud, Jelski decide continuar permaneciendo en América aunque eligiendo un lugar menos tropical y propenso a enfermedades; así, elige Bolivia como destino para seguir sus investigaciones. Los caminos de Jelski y Raimondi se cruzaron casualmente en 1869 y se prolongaron por espacio de casi veinte años.

Estando en travesía hacia Bolivia, Jelski conoce a Eduardo de Habich, ingeniero polaco quien se encontraba en dirección a Lima, contratado por el gobierno peruano (López Soria, 1999: 111), quien lo convence para permanecer algunos días en la ciudad. Jelski aprovechó su estadía en Lima para tomar contacto con Raimondi y en sus propias palabras “... casi sin darme cuenta me quedé en este país ...” ([Kochanek], 1979: 108). Jelski permaneció en el Perú por espacio de nueve años, cinco de ellos con el apoyo del Conde Branicki. En esos primeros años siguió enviando colecciones zoológicas y botánicas al Gabinete de Varsovia y otras de minerales a la Universidad de Cracovia. En 1874, tras aceptar la propuesta que le formula Raimondi para custodiar el Museo Natural que venía formándose en Lima — puesto que demandaba continuos viajes para incrementar las colecciones- Jelski comunica a Branicki la necesidad de enviar a otra persona en su reemplazo. Así, en 1875, Jan Sztolzman, con estudios de ornitología en la Universidad de Varsovia, llegó al Perú a hacerse cargo de las funciones que venía desempeñando Jelski. Como mentor del joven científico polaco — había nacido en Varsovia en 1854- Jelski lo acompañó en varias expediciones viajando por todo el Perú, especialmente por el norte, en los cuales lograron acopiar abundantes colecciones de diversas especies animales no sólo a través de sus propios hallazgos sino también a través de compras de las mismas a nativos de la zona ([Kochanek], 1979: 113). 
Jelski retornó a Polonia en 1878 aceptando el cargo de custodio de las colecciones naturales de la Academia de Ciencias de Cracovia. Su regreso a Polonia no significó cortar los vínculos con Raimondi. Varias cartas dan cuenta de la cordialidad que seguía vigente entre ambos. Sabedor de la seriedad científica de Raimondi, le envía numerosas cartas (32) que pareciera Raimondi no llegó a contestar debido a las dificultades provocadas por la Guerra del Pacífico. Transcurrida esta, la comunicación se restablece. A pesar de desempeñar un puesto importante recibiendo el beneficio del mecenazgo de los condes Branicki, Jelski le solicita un empleo en el Perú como colector pues le será más útil a Raimondi pues “... hice un progreso notable en colectar fósiles ...” y haber aumentado sus conocimientos en botánica y geología (33).

Raimondi también mantuvo comunicación con Sztolzman y con Wladizlaw Taczanowski, célebre ornitólogo polaco que desarrollaba sus actividades en el Museo Zoológico de Varsovia. Raimondi siguió enviando colecciones a Polonia y Sztolzman le sugiere el modo de enviarlas con el mayor cuidado, describiendo pormenorizadamente cada paso para garantizar un embalaje adecuado de las aves (34). Dichas colecciones fueron la base del estudio que Taczanowski dedicó a las aves peruanas, la primera monografía dedicada a aves sudamericanas (Taczanowski, 1884-1886). Otros animales conformaron valiosas colecciones. Del mismo modo, gracias a las colecciones suministradas por Raimondi junto a las que recogieron Jelski y Sztolzman en el Perú, Taczanowski escribió trabajos pioneros sobre arácnidos.

El interés de los científicos peruanos por la riqueza de fauna peruana no se agotó en los casos mencionados. Siempre bajo el patronazgo de los Branicki, el museo organiza una nueva expedición al Perú en 1889. Esta vez, Stolzman comunica que el encargado de realizarla sería el joven "... Kalinowski que ha tenido experiencia en exploraciones por Asia ...”. Iba con el encargo de explorar la parte sur del Perú y seguir a Bolivia habiéndosele sugerido “... que siga el mismo paralelo de exploración desde el mar al Este. Podría ser por Carabaya ...” (35). También fueron numerosas y valiosas las colecciones recolectadas por Jan Kalinowski en su recorrido de medio siglo por el Perú pero a diferencia de sus dos ilustres predecesores, nunca regresó a Polonia asentándose en la zona de Quincemil, en el Cuzco ([Kochanek], 1979: 116).

Larga es la lista de científicos europeos con los que Raimondi intercambió información. Uno solo de los varios casos de contactos en Europa —señalados por Janni (1942: 283) —, fue el que mantuvo con el ingeniero Conde Ferdinand de Lesseps, presidente de la Sociedad de Geografía de París quien lo invita en 1889 a formar parte del Patronato del Congreso Internacional de Ciencias Geográficas que se llevaría a cabo en dicha ciudad (Raimondi, 1990: 68). Raimondi ya formaba parte de dicha

(32) Museo Antonio Raimondi (Lima). Archivo Raimondi. Epistolario. Constantino Jelski a Antonio Raimondi. Cracovia, 5 de noviembre de 1880.

(33) Museo Antonio Raimondi (Lima). Archivo Raimondi. Epistolario. Constantino Jelski a Antonio Raimondi. Sin fecha.

(34) Museo Antonio Raimondi (Lima). Archivo Raimondi. Epistolario. Jan Sztolzman a Antonio Raimondi. Varsovia, 4 de abril de 1882.

(35) Museo Antonio Raimondi (Lima). Archivo Raimondi. Epistolario. Jan Sztolzman a Antonio Raimondi. Varsovia, 20 de junio de 1889. 
asociación; el mismo Lesseps, en calidad de Presidente de la Société de Géographie de Paris - que dirigió entre 1881 y 1889 - lo nombra miembro correspondiente (36). Un año antes se le había librado la acreditación correspondiente. Raimondi contestó entusiastamente la invitación de Lesseps aunque no sabemos si dejó de acudir por problemas económicos o debido a un empeoramiento de su ya frágil salud.

Otra renombrada institución europea a la que se vinculó Raimondi fue la Royal Geographical Society de Londres. A mediados de la década de 1850 publicó en los Proceedings que sacaba a luz dicha corporación científica, dos artículos sobre la zona de Carabaya en Puno, al sur del Perú. No debió ser poca la atención que sus Apuntes sobre la provincia litoral de Loreto, publicada en Lima en 1862, suscitaron en los círculos científicos ingleses. Algo parecido había ocurrido en Francia. Aprovechando las páginas del prestigioso Bulletin de la Société de Géographie, Ernest Desjardin arremetió contra Raimondi, originando la respuesta inmediata de Manuel Rouaud y Paz-Soldán, científico peruano que por entonces se hallaba en Francia coordinando la publicación de las obras de sus célebres tíos, Mateo y Mariano Felipe Paz Soldán.

Por aquel entonces, no existía obra alguna que sintetizara, a partir de una estadía, la realidad de tan alejada zona del país. Debieron ser estos trabajos los que influyeron para que dicha institución lo nombre Miembro Honorario Correspondiente en 1863 (37). Para los ingleses dicho nombramiento abría la posibilidad de seguir aproximándose a regiones que les resultaban desconocidas. Clements Markham, secretario de la sociedad, le comunica las razones de su nombramiento, indicándole que “... tenemos la seguridad que Ud. será un correspondiente y que en consecuencia enviará sus memorias, especialmente sobre el Titicaca y otras vinculadas al Amazonas ..." (38).

De sus contactos epistolares con científicos europeos recibió bibliografía actualizada y abundantes opiniones sobre las especies que solía remitir con frecuencia a Europa. Las colecciones incrementaron el conocimiento en distintas áreas de la ciencia. Por citar un brevísimo ejemplo, tras su deceso, su herbario fue analizado en el más importante centro de conocimiento de la botánica mundial, el Jardín Botánico de Berlín.

José Balta, ingeniero y uno de los más importantes biógrafos del sabio italiano ha hecho la evaluación más ponderada de su contribución a la ciencia al afirmar que “... el carácter distintivo de la obra raimondiana es la acumulación de hechos, datos, limitando las hipótesis y explicación de los fenómenos observados a los estrictamente necesarios ..." (Balta, 1926: 14). Mientras en algunas áreas se ha discutido el valor novedoso de su aporte como en la botánica, excepción hecha de su calidad como herborizador, entendiéndosele como "... simplemente aficionado a la botánica..." (Herrera, 1935: 275) en otras, como en la química, se afirma que “... no hay menor

(36) Antonio Raimondi (Lima). Archivo Raimondi. Diplomas. Société de Géographie de Paris. Membre Correspondant Étranger. París, 11 de mayo de 1888.

(37) Antonio Raimondi (Lima). Archivo Raimondi. Diplomas. Royal Geographical Society (London). Dice el diploma: “... In order to mark the high estimation, constitute him a Foreign Honorary Corresponding Member of the Society...". Londres, 14 de diciembre de 1863.

(38) Antonio Raimondi (Lima). Archivo Raimondi. Epistolario. Clements Robert Markham a Antonio Raimondi. Londres, 15 de diciembre de 1863. 
duda de que Raimondi ha sido el más completo analista, dentro del campo de la química, que haya actuado en el Perú ...” (Guevara, 1993: 135) o la geología, ya que “... pudo reconocer por primera vez la variedad de las formaciones que se presentan en la cordillera ..." (Steinman, 1930: IV).

El proyecto editorial de Raimondi abarcaba seis grandes áreas: geografía, geología, mineralogía, botánica, zoología y etnología (Raimondi, 1874-1879, Tomo III: 61). Dentro de la geografía consideraba el estudio de la meteorología, área que a diferencia de todas las demás no ha recibido atención preferente y cuyo valor es destacabilísimo pues en la exposición de los métodos que empleó para realizar las mediciones de temperatura y presión, aparte de la enorme cantidad de registros practicados en todo el territorio, se revela su decisiva contribución a la ciencia (Raimondi, 1874-1879, Tomo III: 72).

En suma, la intensa vida que Raimondi desplegó en sus cuarenta años en el Perú lo condujo, a partir de su desengaño de la política, por un despliegue docente notable pero frecuentemente discontinuo, el establecimiento de sólidas relaciones amicales y profesionales con las nuevas generaciones de profesionales liberales - pareciera que con la prescindencia de abogados - que conformaban la élite intelectual limeña, una fatigante pero fructífera relación con el Estado peruano y un permanente establecimiento y mantenimiento de redes científicas en América y Europa, actividades todas que conformaron una suerte de contexto en el cual pudo desplegar su talento científico y plasmar su decisiva contribución a la promoción y desarrollo de las ciencias naturales en el Perú.

\section{Referencias citadas}

\section{Fuentes de Archivo}

ARCHIVO GENERAL DE LA NACIÓN. EPISTOLARIO DE MANUEL PARDO - 18731876.

MUSEO ANTONIO RAIMONDI (Lima). Archivo Raimondi.

http://museoraimondi.fundaciontelefonica.org.pe/

ALBERTINI, L. E., 1878 - Exposition Universelle. Le Pérou en 1878 ; notice historique et statistique suivie du catalogue des exposants, 47p.; París: Imprimerie Nouvelle.

AZZARITI, V., 2000 - Vida y obra de Antonio Raimondi, 20p.; Lima: Asociación Educativa Antonio Raimondi.

BALTA, J., 1899 - Labor de Raimondi. Boletín de la Sociedad Geográfica de Lima, IX(1, 2, 3): 8-14.

BALTA, J., 1926 - Labor de Raimondi; Lima: Imprenta Torres Aguirre.

BASADRE, J., 1983 - Historia de la República del Perú; Lima: Ediciones Euroamericanas. $7^{\text {a }}$ Edición.

BONFIGLIO, G., 1993 - Los italianos en el Perú, 334p.; Lima: Saywa.

CUETO, M., 1993 - Excelencia científica en la periferia; actividades científicas e investigación biomédica en el Perú, 1890-1950, 230p.; Lima: Grade. 
CUETO, M., 1995 - Guía para la historia de la ciencia: archivos y bibliotecas en Lima. In: Saberes andinos; ciencia y tecnología en Bolivia, Ecuador y Perú (M. Cueto, ed.): 159213; Lima: IEP.

EL SIGLO, 1874 - (Lima).

FUENTES, M. A., 1858 - Estadística general de Lima, 774p.; Lima: Tipografía de Manuel Nicolás Corpancho.

GUEVARA, J. de D., 1993 - Historia de la Química en el Perú, 492p.; Lima: CONCYTEC.

HERRERA, F., 1935 - Antonio Raimondi, su labor botánica. Sobretiro de la Revista del Museo Nacional: 267-281.

HUMBOLDT, A. von, 1814-1831 - Relation historique du voyage aux régions équinoxiales du Nouveau Continent, fait en 1799, 1800,1801, 1802, 1803 et 1804; París-Londres: À Paris chez F. Schoell, N. Maze; À Londres chez J. Smith.

JANNI, E., 1942 - Vida de Antonio Raimondi, 322p.; Lima: Imp. T. Scheuch.

JUARRANZ DE LA FUENTE, J. M., 1984 - Las revoluciones de 1848, 64p.; Madrid: Akal.

[KOCHANEK, K.], 1979 - Los polacos en el Perú, 200p.; Lima: Embajada de Polonia en el Perú.

LA TORRE, R., 2000 - La obra de Antonio Raimondi en el desarrollo nacional. Boletín de la Sociedad Geográfica de Lima, 113: 203-222.

LLONA, E., 1884 - La obra de Raimondi, 115p.; Lima: Imprenta de Peter Bacigalupi y Ca.

LÓPEZ SORIA, J. I., 1999 - Historia de la Universidad Nacional de Ingeniería. Los años fundacionales (Tomo I), 303p.; Lima: Proyecto Historia UNI.

LÓPEZ SORIA, J. I. \& CAZORLA, I., 1999 - Malinowski, el ingeniero de los ferrocarriles, 61p.; Lima: Proyecto Historia UNI.

MINGUET, C. (ed.), 1980 - Cartas Americanas/ Alejandro de Humboldt, 428p.; Caracas: Biblioteca Ayacucho.

OVIEDO, J., 1862 - Colección de leyes, decretos y órdenes publicadas en el Perú desde el año de 1821 hasta el 31 de diciembre de 1859, Tomo IX; Lima: Felipe Bailly.

PALACIOS, R., 2002 - La Sociedad Geográfica de Lima; fundación y años iniciales, 78p.; Lima: Universidad de Lima, $2^{\mathrm{a}}$ edición.

PALMA, R., 1910 - Discurso en la inauguración del monumento a Antonio Raimondi. El Comercio, 15 de agosto.

PHILIPPI, [R.], 1857 - Noticias zoológicas relativas a la fauna chilena. Anales de la Universidad de Chile. Primera serie: $179-187$.

RAIMONDI, A., 1854 - Informes sobre la existencia de guano en las islas de Chincha presentados por la Comision nombrada por el gobierno peruano, con los planos levantados por la misma Comision, 52p.; Lima: Tipografia de "El Heraldo".

RAIMONDI, A., 1856 - Mémoire sur le guano et les oiseaux qui le produisent (extrait). Comptes Rendus hebdomadaires des séances de l'Académie des Sciences, 42: 735-738; París.

RAIMONDI, A., 1864 - Análisis de las aguas termales de Yura, aguas minerales de Jesús y aguas potables de Arequipa, 16p.; Arequipa: Imprenta de Francisco Ibáñez.

RAIMONDI, A., 1873a - El departamento de Ancachs y sus riquezas minerales, 651p.; Lima: El Nacional.

RAIMONDI, A., 1873b - La manipulación del guano, 61p.; Lima: Imprenta del Estado.

RAIMONDI, A., 1873c - Manipulación del guano, 29p.; Lima: Imprenta de "El Nacional".

RAIMONDI, A., 1874-1879 - El Perú. Lima: Imprenta del Estado.

RAIMONDI, A., 1878 - Minerales del Perú o catálogo razonado de una colección que representa los principales tipos minerales de la república, con muestras de huano y restos de aves que lo han producido, 305p.; Lima: Imprenta del Estado. 
RAIMONDI, A., 1878 - Minéraux du Pérou. Catalogue raisonné d'une collection des principaux types minéraux de la République comprenant aussi des échantillons de guano et des derbis fossilisés des oiseaux qui l'ont produit, 356p.; París: Imp. Centrale des Chemins de fer A. Chaix et Cie.

RAIMONDI, A., 1990 - Epistolario de Antonio Raimondi, 95p.; Lima: Asociación Educacional Antonio Raimondi.

RAIMONDI, A., 1991 - Apreciaciones personales: Cartas a Miguel Colunga (1859-1868), 196p.; Lima: Biblioteca Nacional del Perú.

ROSPIGLIOSI VIGIL, C., 1938 - Historia de los museos en el Perú. Boletín del Museo de Historia Natural, 4: 1-17.

SANTILLANA, T., 1989 - Los viajes de Raimondi, 222p.; Lima: OXY.

SCHELL, P. - The Philippi family in Chile. In: Relics and Selves (interactive exhibition) Iberoamerican Museum of Visual Culture on the web. Birkbeck College. University of London. http://www.bbk.ac.uk/ibamuseum/texts/Schell04A.htm

SEINER, L., 2003 - Antonio Raimondi: difusor pionero de la ciencia en el Perú. Revista de la Facultad de Lenguas Modernas, 6: 155-170; Lima: Universidad Ricardo Palma.

STEINMANN, G., 1930 - Geología del Perú, 448p.; Heidelberg: Carl Winters universität sbuchhklung.

TACZANOWSKI, L., 1884-1886 - Ornithologie du Pérou; Rennes: Typographie Oberthur. 3 vol.

UEDA TSUBOYAMA, M., 2002 - Historia del Cuerpo de Ingenieros de Minas del Perú, 19021950, 252p; Lima: Proyecto Historia UNI.

VALDIZÁN, H., 1924 - Los médicos italianos en el Perú; Lima: Tip. R. Varese. 